\title{
A FURTHER NOTE ON FLEXIBLE LEAST SQUARES AND KALMAN FILTERING
}

\author{
R. KALABA and L. TESFATSION \\ University of Southern California, Los Angeles, CA 90089, USA
}

Received September 1989

Tucci (1990) logically errs when he attempts to equate the flexible least squares (FLS) approach [Kalaba and Tesfatsion (KT) (1989)] with Kalman filtering. FLS addresses a multicriteria model specification problem which does not require probability assumptions either for its motivation or for its solution: the characterization of the set of all state sequence estimates which achieve vector-minimal incompatibility between imperfectly specified theoretical relations and process observations. Kalman filtering is a point estimation technique for determining the most probable state sequence estimate for a stochastic model assumed to be correctly and completely specified.

To illustrate, consider the simple time-varying linear regression problem analyzed both in KT (1989) and in Tucci (1990). Scalar observations $y_{1}, \ldots, y_{T}$ have been obtained on an economic process which is not yet well understood. For simplicity, a linear relation is postulated between the observation $y_{t}$ and a vector $x_{t}$ of given regressor variables at each time $t$, of the form $y_{t} \approx x_{t}^{\prime} b_{t}$, $t=1, \ldots, T$. Although it is recognized that some systematic time variation in the coefficient vectors $b_{t}$ might have occurred over the observation period, it is anticipated that such evolution will have been gradual, so that the successive coefficient vectors satisfy $b_{t+1} \approx b_{t}, t=1, \ldots, T-1$. Each possible estimate $\hat{b}=\left(\hat{b}_{1}, \ldots, \hat{b}_{T}\right)$ for the sequence of coefficient vectors thus entails two conceptually distinct types of model specification errors: namely, measurement errors consisting of the discrepancies $\left[y_{t}-x_{t}^{\prime} \hat{b}_{t}\right]$ between the observation and the estimated linear regression at each time $t$, and dynamic errors consisting of the discrepancies $\left[\hat{b}_{t+1}-\hat{b}_{t}\right]$ between the estimated coefficient vectors at successive times $t$ and $t+1$.

The FLS approach acknowledges the multicriteria nature of this estimation problem, and the minimal nature of the available information concerning the true underlying process. A measurement $\operatorname{cost} c_{M}(\hat{b})$ and a dynamic cost $c_{D}(\hat{b})$ are separately assessed for the two disparate types of model specification errors entailed by the choice of a coefficient sequence estimate $\hat{b}$. Letting $C$ denote the collection of cost vectors $\left(c_{D}(\hat{b}), c_{M}(\hat{b})\right)$ corresponding to all 
possible estimates $\hat{b}$, attention is focused on the lower envelope of $C$ - the 'residual efficiency frontier'. The frontier gives all possible efficient tradeoffs between measurement and dynamic costs. In particular, the frontier shows the measurement cost which must be paid in order to achieve the zero dynamic cost (time-constant coefficients) required by OLS estimation. Each estimate $\hat{b}$ whose associated cost vector attains this frontier has the following basic efficiency property: For the given observations, no other possible coefficient sequence estimate can be found which yields a lower level for one type of cost without increasing the level of the other.

On the basis of both tractability and general appeal, the cost assessments $c_{M}(\hat{b})$ and $c_{D}(\hat{b})$ can be specified as sums of the squared measurement and dynamic errors, respectively. The coefficient sequence estimate $\hat{b}(\mu)$ corresponding to any given point $\mu$ on the residual efficiency frontier then solves a problem of the form 'minimize $c_{M}(\hat{b})$ with respect to $\hat{b}$ subject to $c_{D}(\hat{b})=$ constant', and can be derived sequentially by means of recurrence relations which have the familiar Riccati equation form. This is hardly surprising; it has been known for decades [Bellman and Kalaba (1965)] that linear-quadratic minimization leads to recurrence relations of this type.

Tucci (1990) introduces independence and normality restrictions for the model discrepancy terms under which these FLS recurrence relations for generating any particular estimate $\hat{b}(\mu)$ along the frontier can be given a Kalman filter interpretation. More precisely, as previously shown in KT (1988, app. B), under these probability restrictions the FLS recurrence relations reduce to information filter equations, the 'inverse' of Kalman filter equations. If an analyst believes these probability restrictions to be justified, we would by all means agree that they should be incorporated into the FLS procedure. However, Tucci's claim that such probability restrictions are implied by the FLS procedure has no basis whatsoever.

What is interesting is the new twist which FLS gives to the interpretation of Kalman filter estimates. It is now conventional to interpret any quadratic criterion function representing sums of squared measurement and dynamic errors - e.g., the Kalman filter criterion function - as a log-likelihood expression arising from somc underlying stochastic model in which model discrepancy terms are interpreted as independent and normally distributed random variables. Yet it is also well-known that Kalman filtering works remarkably well in contexts in which these strong stochastic assumptions are not even remotely satisfied. A partial explanation for this robustness is that the Kalman filter criterion function can be given an alternative interpretation: namely, as a cost function embodying the criterion that model discrepancy terms be small. 'Smallness' should not be confused with 'randomness'. Postulating that $b_{t+1}$ is close to $b_{t}$ does not mean that the discrepancy term $\left[b_{t+1}-b_{t}\right]$ is necessarily a random vector. As numerous experiments have shown [KT (1989)], the FLS postulate of small changes in the coefficient vectors $b_{t}$ is a powerful assump- 
tion which allows systematic time variations in these vectors (e.g., regime shifts and sinusoidal variations) to be tracked and recovered with surprising qualitative accuracy at each point along the residual efficiency frontier.

\section{References}

Bellman, R. and R. Kalaba, 1965, Dynamic programming and modern control theory (Academic Press, New York, NY).

Kalaba, R. and L. Tesfatsion, 1988, An organizing principle for dynamic estimation, Journal of Optimization Theory and Applications, forthcoming.

Kalaba, R. and L. Tesfatsion, 1989, Time-varying linear regression via flexible least squares, International Journal of Computers and Mathematics with Applications 17, 1215-1245.

Tucci, M., 1990, A note on flexible least squares, Journal of Economic Dynamics and Control, this issue. 